\title{
Complete and Sustained Response with a Doublet Chemotherapy Protocol in an 81-Year-Old Patient with Metastatic Breast Cancer
}

\author{
Taynah Cascaes Puty ${ }^{a, b}$ Gabriel S.A. Brito ${ }^{a, c}$ Mariana S. Dias ${ }^{a, b}$ \\ Henrique C. Miranda $a^{a, b}$ Juliana R. Chaves ${ }^{a, d}$ Heryvelton L. Freitas \\ Luís E.W. Carvalho ${ }^{a, d}$ \\ a Oncológica Brazil - Education and Research, Belém, Brazil; ${ }^{\text {} U n i v e r s i d a d e ~ d o ~ E s t a d o ~ d o ~}$

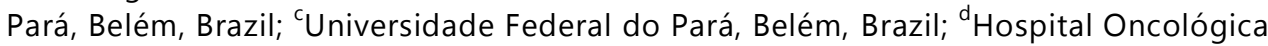 \\ Brasil, Belém, Brazil; ${ }^{\mathrm{e}}$ Hospital Porto Dias, Belém, Brazil
}

\section{Keywords}

Doublet chemotherapy $\cdot$ Breast cancer $\cdot$ Old age $\cdot$ Complete response

\begin{abstract}
Metastatic breast cancer (MBC) entails an overall 5 -year survival of approximately $25 \%$. The choice of therapy is influenced by expression of the HER2 gene and hormone receptors, by a disease-free interval, and by age. The use of paclitaxel combined with gemcitabine (doublet protocol) has shown efficacy as first-line treatment for $M B C$ in either initial or maintenance therapy when compared to monotherapy with paclitaxel. There is evidence showing that the doublet protocol is a good alternative to maintenance therapy in women under 50 years old. Nevertheless, there is a lack of information concerning individuals above that age. We report the case of an 81-year-old patient presenting with recurrence of $M B C$, with lung and skin metastases both positive for hormone receptor and negative for HER2. We implemented a therapy based on the combination of gemcitabine and paclitaxel for 12 cycles, when complete response was achieved. Currently, 16 months after this achievement, the patient is receiving maintenance treatment under the doublet protocol, presenting acceptable parameters of toxicity since the beginning of treatment, which shows satisfactory tolerability and management of chemotherapy in an elderly patient. We suggest that the maintenance
\end{abstract}


treatment protocol with a doublet might be an alternative with a satisfactory response in patients with MBC.

(C) 2016 The Author(s)

Published by S. Karger AG, Basel

\section{Introduction}

Despite advances in diagnosis and therapy, breast cancer is still the leading cause of cancer-related death among women worldwide $[1,2]$. With metastatic disease, overall 5year survival is approximately $25 \%$ [3] and chemotherapy is usually recommended. Howev$\mathrm{er}$, the adequate duration of first-line therapy remains controversial [4]. Expression of HER2 and hormone receptor genes, a disease-free interval, and age are among the factors that influence the choice of first-line therapy in metastatic disease [5, 6].

The use of paclitaxel combined with gemcitabine (doublet protocol) has shown efficacy as first-line initial and maintenance therapy treatment in metastatic breast cancer (MBC) when compared to monotherapy with paclitaxel [7]. There is current evidence that the use of the doublet protocol can also be an interesting alternative to maintenance therapy in women under 50 years old [4]. However, there is almost no information with regard to individuals above that age.

This study reports the case of an 81-year-old woman with aggressive MBC who was treated under a doublet protocol of gemcitabine and paclitaxel as first-line and maintenance treatment after an initial 12 cycles, and she showed complete clinical and radiological response on PET-CT. Despite patients' advanced age and high toxicity described in the literature $[4,7]$, we observed moderate toxicity and improvement in the clinical condition of the patient.

\section{Case Report}

An 81-year-old woman with an ulcerated skin lesion in the right parasternal region measuring $2 \mathrm{~cm}$, as well as multiple satellite injuries in the same area, presented to the Clinical Oncology Service in July 2014. She had several diffusely and bilaterally distributed pulmonary nodules detected by CT, the largest located in the right higher basal segment measuring $1 \mathrm{~cm}$. The patient had a history of malignant neoplasm in the right breast, diagnosed in November 1998 and treated with radical mastectomy, followed by adjuvant chemotherapy with anthracyclines and subsequent adjuvant hormone therapy for 5 years. The anatomical and pathological as well as the immunohistochemical study of the cutaneous lesions confirmed the recurrence of breast cancer, being both positive for hormone receptors and negative for HER2, which was in agreement with a biopsy from the first diagnosis of neoplastic disease.

In July 2014, she underwent control PET-CT (fig. 1a), which showed hypermetabolism in pulmonary nodules [baseline standardized uptake values (SUV) 4.0-5.0], in a right parasternal lesion (SUV 7.7 at baseline), and in left axillary (SUV 4.9 at baseline), right cardiophrenic (SUV 2.0 at baseline), and right retrosternal (SUV 3.0 at baseline) lymph nodes. The chemotherapy protocol with a doublet was started (gemcitabine $1,250 \mathrm{mg} / \mathrm{m}^{2}$ on days 1 and 8 combined with paclitaxel $175 \mathrm{mg} / \mathrm{m}^{2}$ on day 1 every 21 days) and initially planned until progressive disease or limiting toxicity [4]. In addition, the patient underwent 16 sessions of 3D conformal radiotherapy for the right parasternal lesion (dose of 4,000 cGy in a TrueBeam 
STX machine with an energy of $6 \mathrm{mV}$ per dose fraction of $250 \mathrm{cGy}$ ) during 23 days due to metastasis to the anterior chest region (fig. 2).

In October 2014, after the 6th cycle of treatment, thorax and abdomen CT showed a reduction of the mediastinal cardiophrenic lymph nodes and parasternal lesion as well as stability of the pulmonary nodules. In April 2015, in the 12th cycle, a new PET-CT for evolution control showed the disappearance of areas of increased uptake in multiple pulmonary nodules. There was also disappearance of increased uptake in the right parasternal lesion as well as the left axillary, right cardiophrenic, and right retrosternal lymph nodes. There was no appearance of new metabolically active lesions compared with the previous PET-CT, except for the appearance, in the right upper lobe, of a slightly increased uptake (SUV 3.3) suggestive of bronchopneumonic alteration or even secondary to the applied therapy (fig. 1b).

Twelve cycles of first-line treatment with the doublet protocol were carried out from the time of relapse in July 2014. Since April 2015, the patient has received maintenance treatment with the doublet protocol ( 16 cycles thus far) with improvement in performance status and resuming her regular activities. The patient presented grade 2 hematologic toxicity (neutropenia) and grade 3 alopecia. The carcinoembryonic antigen and cancer antigen 15-3 markers remained stable throughout treatment (from 1.8 to $2.8 \mathrm{ng} / \mathrm{ml}$ and from 10 to 20 $\mathrm{U} / \mathrm{ml}$, respectively). Currently, the patient is asymptomatic.

\section{Discussion}

Regarding the treatment and maintenance polychemotherapy used in breast cancer, the use of paclitaxel combined with gemcitabine has shown better results than monotherapy with paclitaxel [8], one of the options recommended as first-line treatment [7]. Currently, there is no consensus on the optimal duration of the doublet protocol for maintenance therapy. A meta-analysis showed improvement in progression-free survival in patients undergoing maintenance chemotherapy (several schemes) until progressive disease or limiting toxicity compared to patients who underwent schemes with a fixed number of cycles [4].

Subsequently, the KCSG-BR07-02 study (phase III) showed that patients who achieved disease control with 6 initial cycles of chemotherapy with paclitaxel plus gemcitabine and received maintenance doses equal to the initial treatment (gemcitabine $1,250 \mathrm{mg} / \mathrm{m}^{2}$ on days 1 and 8 coupled with paclitaxel $175 \mathrm{mg} / \mathrm{m}^{2}$ on day 1 every 21 days) had an average progression-free survival higher than that of the group that was only monitored (7.5 vs. 3.8 months; $p=0.026$ ). The mean overall survival was also higher in the group that received the maintenance scheme (32.3 vs. 23.5 months; $p=0.047$ ), but these findings were obtained from patients younger than 50 years [9]. Our 81-year-old patient presented a better response than what was shown in this phase III study. Currently, she has a disease-free survival of 16 months and an overall survival of 26 months.

The findings of the KCSG-BR07-02 study [9] also draw attention to the high hematological toxicity (grade 3 and 4) resulting from this scheme compared to what was found in the observation group (patients who did not keep receiving treatment until disease progression or who withdrew their consent to participate in the study) (neutropenia 87.1 vs. 30.4\%; p < 0.001 ). In contrast to these findings regarding high toxicity in maintenance treatment, our patient, despite her advanced age, achieved a complete and sustained radiological response in the 12th cycle of treatment with doublet protocol maintenance chemotherapy and so far has had no sign of toxicity higher than grade 2 . 
It is noteworthy that the toxicity found in our patient is considerably less than expected for doublet treatment (mainly during the maintenance phase), even though she was treated with the same dose as the patients in the other studies [9-11]. In addition, our patient had normal anthropometric measurements and absence of sarcopenia, which contributed to the lower toxicity (threshold level 2) [12], since studies show that anthropometric measurements, as well as sarcopenia, function as toxicity indicators in chemotherapy $[13,14]$.

In fact, there is still a lack of studies investigating the risk-benefit relation for maintenance gemcitabine-paclitaxel doublet therapy in older patients with MBC. Our report contributes to answering this question by showing that an elderly patient treated under a prolonged doublet protocol showed increased overall survival without severe toxicity [15].

Therefore, this report reinforces the need for larger studies of maintenance therapy with doublet protocols in patients over 50 years with breast cancer, given the complete and sustained radiological response, increased progression-free survival, and acceptable tolerability found in our patient, as well as considering our patient's age.

\section{Statement of Ethics}

The authors have no ethical conflicts to disclose.

\section{Disclosure Statement}

The authors have no conflicts of interest to disclose.

\section{References}

1 American Cancer Society: Global Cancer Facts and Figures, ed 3. 2015. http://www.cancer.org/acs/groups/content/@research/documents/document/acspc-044738.pdf (accessed August 8, 2015).

2 Jemal A, Bray F, Center MM, Ferlay J, Ward E, Forman D: Global cancer statistics. CA Cancer J Clin 2011;61:69-90.

3 National Cancer Institute: SEER Cancer Statistics Review, 1975-2012. 2015. http://seer.cancer.gov/archive/csr/1975_2012/results_merged/topic_survival.pdf (accessed June 15, 2016).

4 Gennari A, Stockler M, Puntoni M, Sormani M, Nanni O, Amadori D, et al: Duration of chemotherapy for metastatic breast cancer: a systematic review and meta-analysis of randomized clinical trials. J Clin Oncol 2011;29:2144-2149.

5 Sio TT, Chang K, Jayakrishnan R, Wu D, Politi M, Malacarne D, et al: Patient age is related to decisionmaking, treatment selection, and perceived quality of life in breast cancer survivors. World J Surg Oncol 2014;12:230.

-6 Yao ZX, Lu LJ, Wang RJ, Jin LB, Liu SC, Li HY, et al: Discordance and clinical significance of ER, PR, and HER2 status between primary breast cancer and synchronous axillary lymph node metastasis. Med Oncol 2014;31:798.

-7 Albain KS, Nag SM, Calderillo-Ruiz G, Jordaan JP, Llombart AC, Pluzanska A, et al: Gemcitabine plus paclitaxel versus paclitaxel monotherapy in patients with metastatic breast cancer and prior anthracycline treatment. J Clin Oncol 2008;26:3950-3957.

8 Rau KM, Li SH, Chen SM, Tang Y, Huang CH, Wu SC, et al: Weekly paclitaxel combining with gemcitabine is an effective and safe treatment for advanced breast cancer patients. Jpn J Clin Oncol 2011;41:455461.

-9 Park YH, Jung KH, Im SA, Sohn JH, Ro J, Ahn JH, et al: Phase III, multicenter, randomized trial of maintenance chemotherapy versus observation in patients with metastatic breast cancer after achieving disease control with six cycles of gemcitabine plus paclitaxel as first-line chemotherapy: KCSG-BR07-02. J Clin Oncol 2013;31:1732-1739. 


\section{Case Reports in Oncology}

\begin{tabular}{l|l}
\hline Case Rep Oncol 2016;9:580-585 \\
\hline DOI: 10.1159/000449127 & $\begin{array}{l}\text { C 2016 The Author(s). Published by S. Karger AG, Basel } \\
\text { www.karger.com/cro }\end{array}$ \\
\hline
\end{tabular}

Puty et al.: Complete and Sustained Response with a Doublet Chemotherapy Protocol in an 81-Year-Old Patient with Metastatic Breast Cancer

10 Salvador J, Manso L, de la Haba J, Jaen A, Ciruelos E, de Villena MC, et al: Final results of a phase II study of paclitaxel, bevacizumab, and gemcitabine as first-line therapy for patients with HER2-negative metastatic breast cancer. Clin Transl Oncol 2015;17:160-166.

-11 Mastro LD, Fabi A, Mansutti M, Laurentiis MD, Durando A, Merlo DF, et al: Randomised phase 3 openlabel trial of first-line treatment with gemcitabine in association with docetaxel or paclitaxel in women with metastatic breast cancer: a comparison of different schedules and treatments. BMC Cancer 2013;13:164.

12 National Institutes of Health; National Cancer Institute; US Department of Health and Human Services: Common Terminology Criteria for Adverse Events (CTCAE) version 4.0. 2009. http://evs.nci.nih.gov/ftp1/CTCAE/CTCAE_4.03_2010-06-14_QuickReference_8.5x11.pdf (accessed June 15, 2016).

13 Prado CM, Baracos VE, McCargar LJ, Reiman T, Mourtzakis M, Tonkin K, et al: Sarcopenia as a determinant of chemotherapy toxicity and time to tumor progression in metastatic breast cancer patients receiving capecitabine treatment. J Clin Oncol 2009;15:2920-2926.

14 Abraham JE, Hiller L, Dorling L, Vallier AL, Dunn J, Bowden S, et al: A nested cohort study of 6,248 early breast cancer patients treated in neoadjuvant and adjuvant chemotherapy trials investigating the prognostic value of chemotherapy-related toxicities. BMC Med 2015;13:306.

15 Mariano C, Francl M, Pope J, Wong L, Lim HJ, Lohrisch C: Comparison of toxicity experienced by older versus younger patients enrolled in breast cancer clinical trials. Clin Breast Cancer 2015;15:73-79.
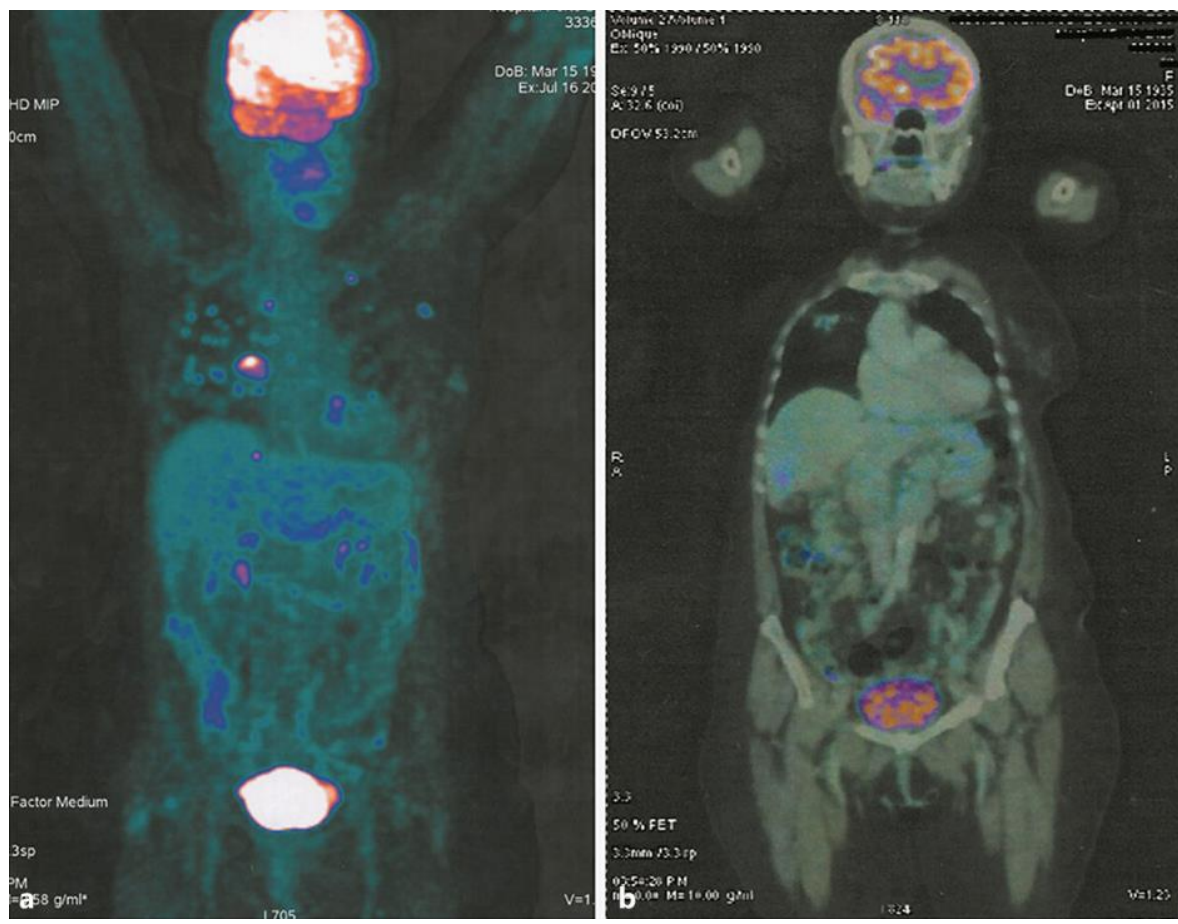

Fig. 1. a PET-CT showing hypermetabolic nodules in the lungs, right parasternal region, and left axillary, right cardiophrenic, and right retrosternal lymph nodes. b Control PET-CT after treatment with the doublet protocol, showing absence of hypermetabolic lesions. 


\section{Case Reports in Oncology}

\begin{tabular}{l|l}
\hline Case Rep Oncol 2016;9:580-585 \\
\hline DOI: 10.1159/000449127 & $\begin{array}{l}\text { c } 2016 \text { The Author(s). Published by S. Karger AG, Basel } \\
\text { www.karger.com/cro }\end{array}$ \\
\hline
\end{tabular}

Puty et al.: Complete and Sustained Response with a Doublet Chemotherapy Protocol in an 81-Year-Old Patient with Metastatic Breast Cancer
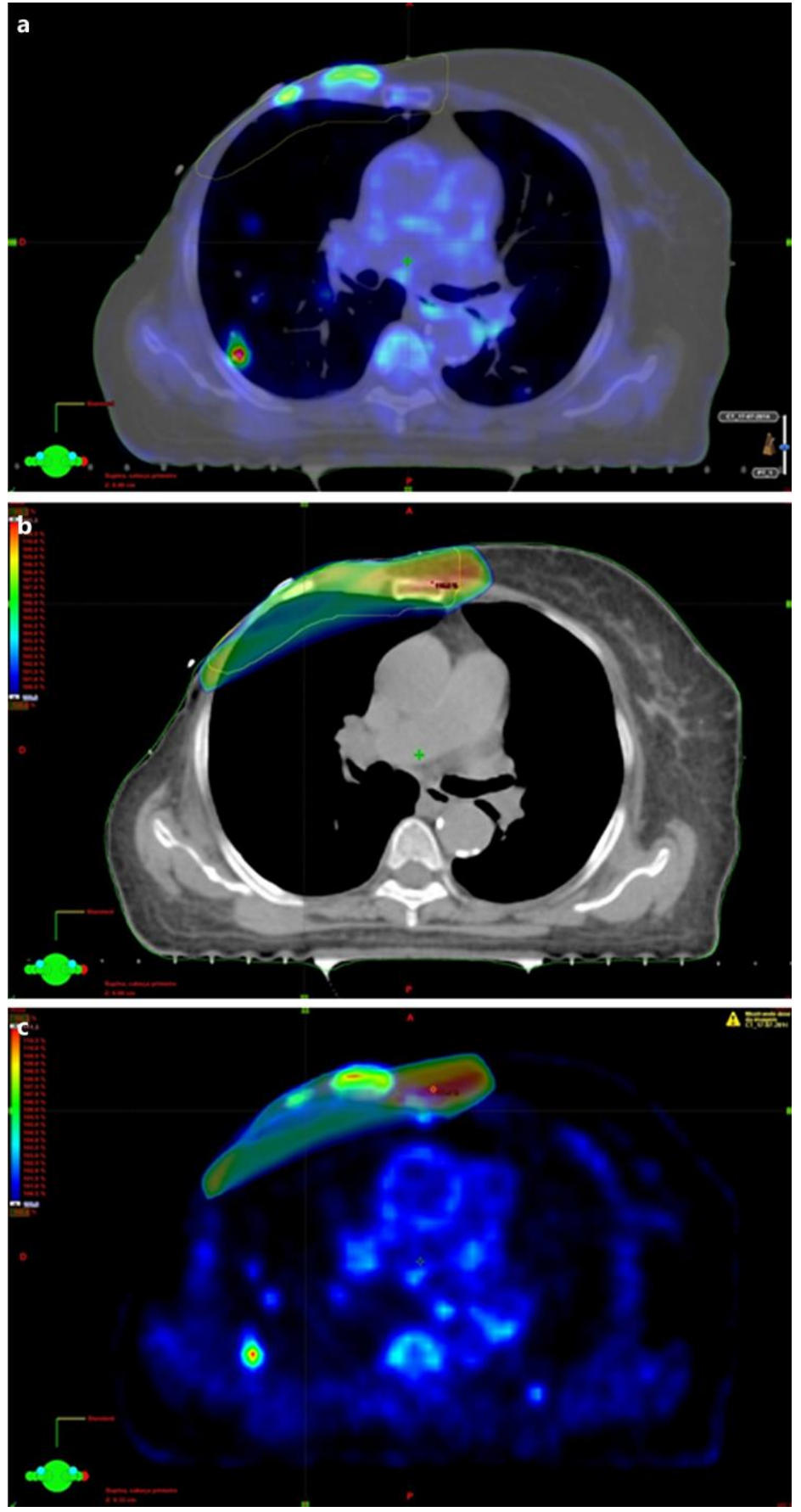

Fig. 2. Image of 3D conformal radiotherapy with a TrueBeam STX machine at $6 \mathrm{mV}$ energy and a dose of 250 cGy of the lesion in the right parasternal region. a PET-CT image. $\mathbf{b}$ Dose image. $\mathbf{c}$ PET image. 